\title{
Changes in Pupil Mydriasis as a Marker for Diabetes
}

\author{
Naveen Kumar Challa ${ }^{1,2 *}$, GO Ovenseri-Ogbomo ${ }^{1,3}$ and Sharma $A^{2}$ \\ ${ }^{1}$ Department of Optometry, College of Applied Medical Sciences, Qassim University, Saudi \\ Arabia \\ ${ }^{2}$ Lotus Eye Care Hospital and Institute, Coimbatore, India \\ ${ }^{3}$ Department of Optometry, University of Benin, Nigeria
}

\section{Research Article \\ Volume 6 Issue 2}

Received Date: September 03, 2021

Published Date: September 27, 2021

DOI: $10.23880 /$ oajo-16000228

*Corresponding author: Naveen Kumar Challa, Department of Optometry, College of Applied Medical Sciences, Qassim University, Saudi Arabia, Tel: 0531177518; Email: N.challa@qu.edu.sa

\section{Abstract}

Clinical Relevance: Optometrists often are the first to examine previously underdiagnosed diabetic patients because of its ocular manifestations such as a change in refractive status. This study is relevant to help optometrists identify patients with diabetes using changes in pupil mydriasis as a marker.

Background: This study compared the pupillary dilatation in subjects with diabetes mellitus and those without diabetes mellitus. The purpose of the study was to use this difference as a diagnostic marker for identification of diabetes in previously undiagnosed subjects who present for eye examination.

Methods: An experimental study was conducted involving 40 non-diabetic and 80 diabetic subjects with or without diabetic retinopathy. Baseline pupil diameter was measured using Orbscan and Aberrometer. Mydriasis was elicited using a combination eye drop (0.8\% Tropicamide and 5\% Phenylephrine). Pupil diameter was measured at 15, 30, 45 and 60 minutes after the instillation of the eye drops. The difference in pupil diameter amongst the three groups was determined using factorial ANOVA to account for the effect of age.

Results: There was no significant difference between the mean pupil diameter among the three groups at baseline when measured with Orbscan $(p=0.187)$ and Aberrometer $(p=0.146)$. There was a significant difference in the mean and mean change in pupil diameter among the three groups with either Orbscan or Aberrometer at 30, 45 and 60 minutes after the instillation of mydriatic drops. The non-diabetics have larger pupil diameter while the diabetics with retinopathy have the least pupil diameter.

Conclusion: The study showed that there was a significant difference in pupil diameter between non-diabetics, diabetics without retinopathy and diabetics with retinopathy during mydriasis. Alongside a history of symptoms of diabetes, this difference could be explored as an ocular biomarker to identify previously undiagnosed diabetes in individuals who present for routine eye examination.

Keywords: Diabetes Mellitus; Mydriasis; Pupil; Diabetic Retinopathy; Hyperglycemia; Aberrometer

\section{Introduction}

Diabetes mellitus is a heterogeneous group of disorders with distinct genetic, etiologic, immunologic and pathophysiologic mechanisms that result in glucose intolerance and hyperglycemia [1]. Given global lifestyle changes, there has been an increase in the prevalence of diabetes globally. An estimated 26 million people were reported to have diabetes in 2010 with an additional 79 million individuals older than 20 years of age with 


\section{Open Access Journal of Ophthalmology}

prediabetes [2]. The increasing prevalence of diabetes mellitus is associated with several risk factors such as aging, physical inactivity, obesity and diet [3].

Diabetes is a multi-systemic disease that often have implications for other organs of the body including the microvasculature of the retina leading to diabetic retinopathy which is one of the leading causes of visual impairment globally. People living with diabetes have a 25 times likelihood of becoming blind compares to normal population [4]. In the US, diabetic retinopathy is responsible for 12,000 to 24,000 new cases of blindness annually raising considerable public health concern [5]. In a Europe-wide study, it was reported that $26 \%$ of patients with microvascular complications of Type 2 diabetes had diabetic retinopathy and other related ocular complications [6]. Besides diabetic retinopathy, other mechanism through which diabetes may cause visual impairment include cataract, glaucoma, nerve palsy and macular degeneration [7].

Prompt and early diagnosis for diabetes mellitus is a critical element in adequate management of diabetes and its associated ocular and other microvascular complications. Several risk factors including pupil diameter, glycated hemoglobin, fructosamine, glycated albumin etc. have been identified and used as biomarkers to predict diabetes and diabetic retinopathy [8-18]. However, these biomarkers have been reported to have inherent limitations. It has been reported that "no single biomarker will also likely have inherent limitations" [11]. They therefore suggested combining several biomarkers to improve early identification of diabetes and diabetic retinopathy.

Individuals presenting for eye examination often may require a dilated funduscopy. Diabetes affects the autonomic nervous system and has been reported to influence pupil diameter. Previous study has reported association between baseline pupil diameter and duration of diabetes and HbA1c [13]. This study was conducted among children aged 6-17 years who have diabetes. The authors used Polaroid photography to measure pupil diameter. In another study it was reported that an inverse relationship between the duration of diabetes and pupil dilation in a South Indian population [19]. In their study vertical pupil diameter was measured at 40 mins post-instillation of mydriatic agent using pupil gauge under bright illumination. There have been contentious reports that there is variation in pupil size between diabetic and non-diabetic subjects. Whereas some authors Karavanaki K, et al. [13], George A, et al. [19] has ported changes in pupil size between diabetic and non-diabetic subjects, others Coblentz J, et al. [20] did not observe such difference in their study. We hypothesized that the contention may be related to the methods employed in the respective study.
The current study was aimed at exploring pharmacologically-induced pupillary dilation as a marker for diabetes. The present study used objective measurement of pupil diameter (Orbscan and Aberrometer) which is more accurate than the manual methods of measuring pupil diameter that was used in the previous study [19]. Furthermore, we explored the temporal effect of mydriasis in non-diabetics and diabetics with and without retinopathy by measuring pupil diameter over a range of time intervals. This study was carried out among Indian population because it has been reported that environmental and genetic factors influence pupillary dilatation [21].

\section{Methods and Materials}

A total of 80 subjects with diabetes mellitus (comprising 40 diabetics without retinopathy and 40 with retinopathy) and 40 controls (who do not have diabetes mellitus) aged 40-80 years who presented to the outpatient department of Lotus Eye Hospital and Institute, Coimbatore, India, were included in the study. All the patients required pupil dilatation and fundus examination. Patients with prior intraocular surgeries, posterior synechiae, shallow anterior chamber, angle closure glaucoma and ocular diseases that could affect the iris, history of hypertension, cardiac problems and injuries were excluded from the study.

The diabetic group was selected based on positive history of diabetes as diagnosed by the attending physician and accompanied by their blood sugar levels. Those with retinopathy were diagnosed based on ophthalmologic examination. All the subjects' diabetic history includes duration of diabetes, recent blood sugar levels, and current mode of treatment.

The diameter of the pupil was measured before dilatation to obtain baseline pre-mydriatic pupil diameter in all subjects in a dark room using Aberrometer (Zyoptix, Bausch \& Lomb) first and then followed by OrbscanIIz (Bausch \& Lomb). This is to avoid the effect of light exposure caused by Orbscan. After the subject is dark adapted for two minutes, pupil diameter is evaluated using aberrometer with monocular vision. Aberrometer uses infrared light with fixation target and measures digitally the pupil diameter using the distributed pattern of the reflected wave front using Hartmann-Shack principle. After aberrometer readings were taken the subject is shifted to orbscan instrument situated in the same room and instructed to fix his monocular viewing to the central part of the placidos disc. OrbscanIIz uses combination of placidos disc and scanning slit technology and uses series of light slits passing over the cornea at angle of $45^{\circ}$ to the camera and records mesopic pupil diameter. This was followed by the instillation of Tropicamide $(0.8 \%)$ and Phenyephrine (5\%). While instilling the drops, the examiner 


\section{Open Access Journal of Ophthalmology}

digitally depressed the nasal canthus of the patient so as to occlude the punctum and prevent the systemic uptake of the eye drops. This would reduce the complications associated with Phenylephrine. After instillation, pupil diameter was measured at 15, 30, 45 and 60 minutes using Orbscan and Aberrometer in a dark room. Three readings were taken at each time interval and the average of the three readings was used for analysis. All the measurements were performed one individual between $11 \mathrm{am}$ and $1 \mathrm{pm}$ each session. This was done to avoid inter-examiner bias and diurnal variation. Pupil diameter was reported as mean \pm SD. The SmirnoffKomogorov test of normality was used to assess the normality of the pupil diameter. The unity-based normalization (minmax normalization) was performed to ensure that the data set was normal. Levene test of homogeneity of variance was performed to assess the homogeneity of variance among the groupsas this was the condition for performing factorial analysis of variance. The $3 \times 4$ (diabetes status $\mathrm{x}$ age group) factorial analysis of variance (ANOVA) was used to compare the pupil diameter among the three groups (nondiabetics and diabetics with and without retinopathy) and the Bonferroniposthoc correction was used to determine the difference between each group. Pearson's correlation coefficient was used to compare the relationship between pupil diameter and age. Furthermore, to account for the effect of age on pupil diameter, the measured pupil diameters at different time intervals were normalized to the baseline using the expression [22].

Change in pupil diameter at time $(\mathrm{t})=$ pupil diameter at time $(\mathrm{t})$ - baseline pupil diameter at time $(\mathrm{t}=0)$. 24.0

All data analyses were performed using the SPSS version

\begin{tabular}{|c|c|c|c|}
\hline Group & Variable & Range & Mean \pm SD \\
\hline \multirow{3}{*}{$\begin{array}{c}\text { Diabetic patients without } \\
\text { retinopathy }\end{array}$} & Age (years) & $40-80$ & $58.75 \pm 9.61$ \\
\cline { 2 - 4 } & Duration of diabetes (years) & $0.1-23$ & $6.55 \pm 5.39$ \\
\cline { 2 - 4 } & Pupil diameter measured with Orbscan (base line) (mm) & $2.9-5.0$ & $4.03 \pm 0.55$ \\
\cline { 2 - 4 } & Pupil diameter measured with Aberrometer (baseline) (mm) & $4.75-7.05$ & $6.07 \pm 0.51$ \\
\hline \multirow{3}{*}{$\begin{array}{c}\text { Diabetic patients with } \\
\text { retinopathy }\end{array}$} & Age (years) & $45-74$ & $59.95 \pm 8.29$ \\
\cline { 2 - 4 } & Duration of diabetes (years) & $0.5-27$ & $8.91 \pm 6.01$ \\
\cline { 2 - 4 } & Pupil diameter measured with Orbscan (base line) (mm) & $3.00-4.90$ & $3.83 \pm 0.51$ \\
\cline { 2 - 4 } & $4.75-6.90$ & $5.72 \pm 0.55$ \\
\hline \multirow{3}{*}{$\begin{array}{c}\text { Non-diabetic patients } \\
n y y y\end{array}$} & Pupil diameter measured with Aberrometer (baseline) (mm) & $40-73$ & $48.68 \pm 7.93$ \\
\cline { 2 - 4 } & Aupil diameter mears) & $3-5.1$ & $4.19 \pm 0.65$ \\
\cline { 2 - 4 } & $4.75-7.75$ & $6.18 \pm 0.74$ \\
\hline
\end{tabular}

Table 1: Baseline data representing the descriptive statistics of all subjects.

The mean duration of diabetes was $7.73 \pm 5.80(95 \%$ CI 6.44-9.02) years. The duration of diabetes ranged from 0.1-27 years. The mean duration for diabetic subjects

\section{Ethical Consideration}

This study was approved by the ethics committee of the Lotus Eye Hospital and Institute, Coimbatore, India. The Lotus Eye Hospital and Institute exist to provide care for patients in an ethical and professional manner. The study was conducted such that patient confidentiality was guaranteed and ensured that no harm was done to study participants as required by the hospital research policy. The study was conducted following the tenets of the Declarations of Helsinki on medical research involving human subjects. All the patients were informed about the study and informed consent was sought and obtained from all eligible participants.

\section{Results}

A total of 120 subjects participated in this study. This comprised of 40 subjects (age range 40-73 years) who did not have diabetes mellitus and 80 subjects (age range 40-80 years) living with diabetes mellitus (comprising 40 subjects each with diabetic retinopathy and no diabetic retinopathy). The mean age of the non-diabetic subjects was $48.68 \pm 7.93$ (95\% CI=46.13-51.21) years while for the diabetic group was $59.35 \pm 8.94$ (95\% CI 57.38-61.32) years. The diabetic group was significantly older than the non-diabetic group $(\mathrm{p}<0.0001)$. The subjects with diabetes but no retinopathy were aged 40-80 years with a mean age of $58.75 \pm 9.61$ years (95\% CI=55.68-61.82). Subjects with diabetes who had diabetic retinopathy were aged 45-74 years with a mean age of $59.95 \pm 8.28$ years $(95 \% \mathrm{CI}=57.30-62.60)$. There was no significant difference between the mean age of subjects with diabetes who had retinopathy and those who do not have retinopathy ( $p=0.552$ ). Table 1 shows the descriptive data of both the diabetic and non-diabetic groups. with no retinopathy was $6.55 \pm 5.39(95 \% \mathrm{CI}=4.83-8.27)$ (range $=0.1-23$ ) years while for the diabetic subjects with retinopathy the mean duration of diabetes was $8.91 \pm 6.01$ \\ 6(2): 000228.}




\section{Open Access Journal of Ophthalmology}

(95\% CI=6.99-10.83) (range=0.5-27) years. There was no significant difference between the mean duration of diabetes in diabetic subjects with or without retinopathy ( $p=0.068$ ).

There was a significant low to moderate negative correlation between pupil diameter and duration of diabetes at baseline and at the different time intervals after instillation of the mydriatic eye drop ( $\mathrm{r}=-0.245$ at 15 mins post-instillation to -0.639 at 45 mins post-instillation using Orbscan and $\mathrm{r}=-0.245$ at 15 mins post-instillation to -0.559 at 45 mins post instillation using Aberrometer). At baseline, there was significant moderate negative correlation between pupil diameter and age of study subjects using Orbscan ( $\mathrm{r}=-$ 0.508, $\mathrm{p}<0.0001)$ and Aberrometer $(\mathrm{r}=-0.424, \mathrm{p}<0.0001)$.

The Smirnoff-Kolmogorov test indicated that pupil diameter was not normally distributed $(p<0.001)$. At baseline, the mean pupil diameter for the non-diabetic subjects was $4.19 \pm 0.65 \mathrm{~mm}$ (Orbscan) and $6.18 \pm 0.74 \mathrm{~mm}$
(Aberrometer). For the diabetic subjects, the mean pupil diameter was $3.93 \pm 0.54 \mathrm{~mm}$ (Orbscan) and $5.90 \pm 0.55 \mathrm{~mm}$ (Aberrometer). For all the study participants, there was a significant difference in the pupil diameter measured with Orbscan compare to values measured with Aberrometer at baseline and at 15 and 30 mins $(\mathrm{p}<0.0001)$ after instillation of mydriatic eye drop. However, this difference was not significant at $45 \mathrm{mins}(\mathrm{p}=0.111)$ and $60 \mathrm{mins}(\mathrm{p}=0.822)$ postinstillation.

For diabetic subjects without retinopathy, the mean pupil diameter at baseline was $4.03 \pm 0.55 \mathrm{~mm}$ (Orbscan) and $6.07 \pm 0.51 \mathrm{~mm}$ (Aberrometer). For diabetic subjects with retinopathy, the mean pupil diameter at baseline was $3.83 \pm 0.51 \mathrm{~mm}$ (Orbscan) and $5.72 \pm 0.55 \mathrm{~mm}$ (Aberrometer). (Figures $1 \& 2$ ) shows the mean pupil diameter measured at baseline and the different time intervals using the Orbscan and Aberrometer respectively for non-diabetic, diabetic with no retinopathy and diabetic with retinopathy.

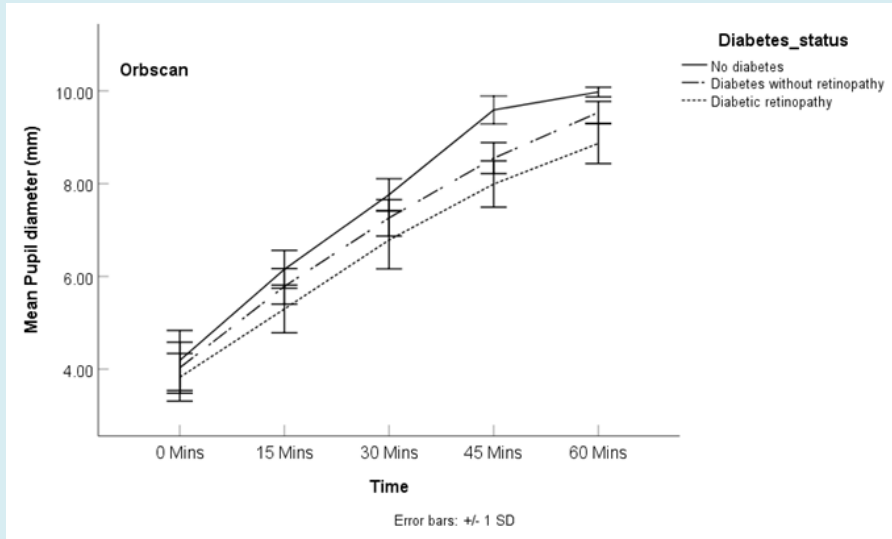

Figure 1: Comparison of mean pupil diameter over the time interval as measured using Orbscan(Error bar represents \pm 1 standard deviation).

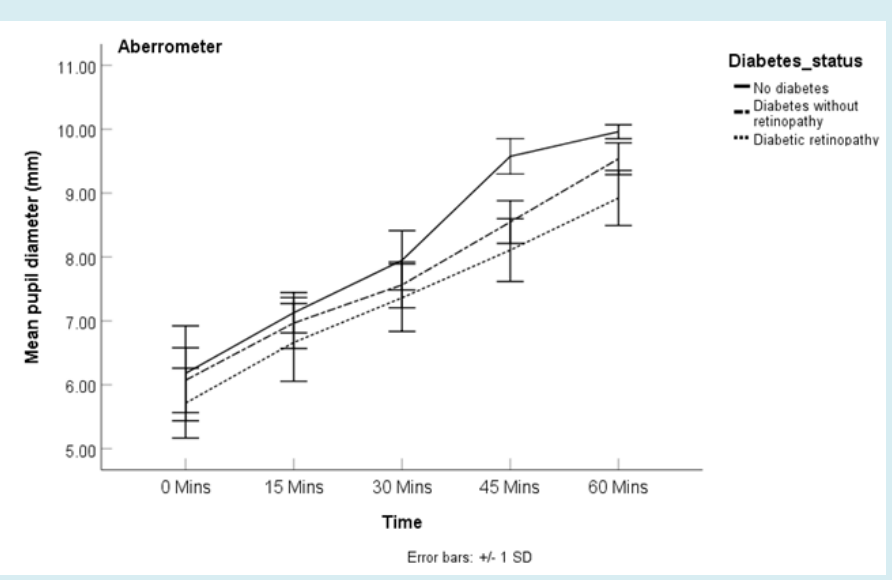

Figure 2: Comparison of mean pupil diameter over the time interval as measured using Aberrometer (Error bar represents \pm 1 standard deviation). 
The mean pupil diameter at baseline and at each time interval after the instillation of mydriatic agent for nondiabetics, diabetics with no retinopathy and diabetics with retinopathy as measured using the Orbscan and the Aberrometer as well as the mean change in pupil diameter from baseline is shown in Table 2. The Levene test of homogeneity of variance indicated that the variances among the groups was homogeinous $(\mathrm{p}=0.606)$. A $3 \times 4$ (diabetes status $x$ age group) factorial analysis of variance (ANOVA) performed on the normalized data indicated that there was a significant difference in pupil diameter among the three groups irrespective of the instrument used in measuring the pupil diameter. At baseline, there was no significant difference among the three groups with either instrument $(\mathrm{p}=0.187$ and $p=0.146$ for Orbscan and aberrometer respectively. At 15 minutes after the instillation of the mydriatic drops, there was a significant difference among the three groups as revealed by the $3 \times 4$ (diabetes status $x$ age group) factorial ANOVA
(Table 2). This difference persisted throughout the duration of the mydriasis. The partial eta squared $\left(\eta^{2}\right)$ value indicated that differences in diabetic status was responsible for 3.1\%, $25.7 \%, 24.9 \%, 55.7 \%$ and $57.5 \%$ of the variation in pupil diameter at baseline, 15, 30, 45 and 60 minutes respectively after the instillation of the mydriatic agent when measured with Orbscan. Over the same time interval, age accounted for 19.4\%, 10.4\%, 11.9\%, 17.3\% and 8.6\% respectively.. Similar to the Orbscan values, when measured with the aberrometer, significant difference in pupil diameter was observed at 15 minutes after instillation of the mydriatic agent and this persisted up to 60 minutes post-instillation. The partial eta squared $\left(\mathrm{n}^{2}\right)$ indicated that the difference in diabetic status accounted for $3.5 \%, 8.7 \%, 6.2 \%, 47.1 \%$ and $51.7 \%$ of the variation in pupil diameter measured with Aberrometer for each of the time interval starting at baseline respectively while age accounted for $14.1 \%, 1.0 \%, 6.2 \%, 9.5 \%$ and $4.9 \%$ respectively (Table 3 ).

a) Orbscan

\begin{tabular}{|c|c|c|c|c|c|c|c|}
\hline $\begin{array}{c}\text { Diabetes } \\
\text { Status }\end{array}$ & $\begin{array}{c}\text { Age } \\
\text { Group }\end{array}$ & $\begin{array}{c}0 \text { Mins } \\
(\text { Mean } \pm \text { SD) }\end{array}$ & $\begin{array}{c}15 \text { mins } \\
(\text { Mean } \pm \text { SD })\end{array}$ & $\begin{array}{c}30 \text { mins } \\
(\text { Mean } \pm \text { SD) }\end{array}$ & $\begin{array}{c}45 \text { mins } \\
(\text { Mean } \pm \text { SD) }\end{array}$ & $\begin{array}{c}60 \text { mins } \\
(\text { Mean } \pm \text { SD })\end{array}$ & $\mathbf{N}$ \\
\hline \multirow{10}{*}{ No diabetes } & $40-49$ & $4.45 \pm 0.49$ & $6.20 \pm 0.43$ & $7.82 \pm 0.36$ & $9.72 \pm 0.22$ & $9.99 \pm 0.04$ & 26 \\
\hline & & & $1.76 \pm 0.53$ & $3.37 \pm 0.54$ & $5.28 \pm 0.46$ & $5.55 \pm 0.48$ & 26 \\
\hline & 50- 59 & $3.94 \pm 0.63$ & $6.10 \pm 0.34$ & $7.73 \pm 0.25$ & $9.44 \pm 0.25$ & $10.00 \pm 0.00$ & 10 \\
\hline & & & $2.16 \pm 0.69$ & $3.79 \pm 0.74$ & $5.50 \pm 0.76$ & $6.06 \pm 0.63$ & 10 \\
\hline & $60-69$ & $3.17 \pm 0.29$ & $6.07 \pm 0.45$ & $7.67 \pm 0.06$ & $9.20 \pm 0.10$ & $9.93 \pm 0.12$ & 3 \\
\hline & & & $2.90 \pm 0.52$ & $4.40 \pm 0.26$ & $6.03 \pm 0.30$ & $6.77 \pm 0.40$ & 3 \\
\hline & $70-79$ & $3.00 \pm 0.00$ & $5.60 \pm 0.00$ & $7.00 \pm 0.00$ & $8.80 \pm 0.00$ & $9.40 \pm 0.00$ & 1 \\
\hline & & & $2.60 \pm 0.00$ & $4.00 \pm 0.00$ & $5.80 \pm 0.00$ & $6.40 \pm 0.00$ & 1 \\
\hline & Total & $4.19 \pm 0.65$ & $6.15 \pm 0.41$ & $7.76 \pm 0.34$ & $9.59 \pm 0.30$ & $9.98 \pm 0.10$ & 40 \\
\hline & & & $1.97 \pm 0.65$ & $3.58 \pm 0.65$ & $5.40 \pm 0.57$ & $5.79 \pm 0.62$ & 40 \\
\hline \multirow{10}{*}{$\begin{array}{c}\text { Diabetes } \\
\text { without } \\
\text { retinopathy }\end{array}$} & $40-49$ & $4.14 \pm 0.62$ & $5.98 \pm 0.22$ & $7.34 \pm 0.37$ & $8.70 \pm 0.34$ & $9.56 \pm 0.35$ & 5 \\
\hline & & & $1.84 \pm 0.51$ & $3.20 \pm 0.49$ & $4.56 \pm 0.47$ & $5.42 \pm 0.50$ & 5 \\
\hline & $50-59$ & $4.24 \pm 0.39$ & $5.98 \pm 0.34$ & $7.48 \pm 0.30$ & $8.71 \pm 0.27$ & $9.61 \pm 0.13$ & 15 \\
\hline & & & $1.74 \pm 0.28$ & $3.24 \pm 0.28$ & $4.47 \pm 0.28$ & $5.37 \pm 0.41$ & 15 \\
\hline & $60-69$ & $4.05 \pm 0.57$ & $5.69 \pm 0.35$ & $7.17 \pm 0.37$ & $8.46 \pm 0.30$ & $9.50 \pm 0.30$ & 14 \\
\hline & & & $1.64 \pm 0.29$ & $3,12 \pm 0.37$ & $4.41 \pm 0.43$ & $5.45 \pm 0.34$ & 14 \\
\hline & $70-79$ & $3.37 \pm 0.34$ & $5.35 \pm 0.26$ & $6.87 \pm 0.34$ & $8.23 \pm 0.34$ & $9.42 \pm 0.17$ & 6 \\
\hline & & & $1.98 \pm 0.30$ & $3.50 \pm 0.36$ & $4.87 \pm 0.39$ & $6.05 \pm 0.45$ & 6 \\
\hline & Total & $4.03 \pm 0.55$ & $5.79 \pm 0.38$ & $7.26 \pm 0.39$ & $8.55 \pm 0.33$ & $9.54 \pm 0.24$ & 40 \\
\hline & & & $1.76 \pm 0.33$ & $3.23 \pm 0.36$ & $4.52 \pm 0.39$ & $5.51 \pm 0.45$ & 40 \\
\hline
\end{tabular}


Open Access Journal of Ophthalmology

\begin{tabular}{|c|c|c|c|c|c|c|c|}
\hline \multirow{10}{*}{$\begin{array}{l}\text { Diabetes } \\
\text { retinopathy }\end{array}$} & $40-49$ & $3.90 \pm 0.64$ & $5.32 \pm 0.74$ & $6.82 \pm 0.81$ & $8.03 \pm 0.59$ & $8.92 \pm 0.39$ & 6 \\
\hline & & & $1.42 \pm 0.12$ & $2.92 \pm 0.26$ & $4.13 \pm 0.14$ & $5.02 \pm 0.36$ & 6 \\
\hline & $50-59$ & $4.09 \pm 0.47$ & $5.53 \pm 0.36$ & $7.03 \pm 0.50$ & $8.19 \pm 0.27$ & $8.90 \pm 0.40$ & 13 \\
\hline & & & $1.44 \pm 0.44$ & $2.94 \pm 0.65$ & $4.10 \pm 0.50$ & $4.81 \pm 0.59$ & 13 \\
\hline & $60-69$ & $3.64 \pm 0.43$ & $5.24 \pm 0.44$ & $6.74 \pm 0.60$ & $7.98 \pm 0.45$ & $8.94 \pm 0.40$ & 15 \\
\hline & & & $1.60 \pm 0.35$ & $3.10 \pm 0.54$ & $4.34 \pm 0.45$ & $5.30 \pm 0.44$ & 15 \\
\hline & $70-79$ & $3.63 \pm 0.49$ & $4.92 \pm 0.57$ & $6.33 \pm 0.60$ & $7.55 \pm 0.71$ & $8.57 \pm 0.60$ & 6 \\
\hline & & & $1.28 \pm 0.54$ & $2.70 \pm 0.51$ & $3.92 \pm 0.72$ & $4.93 \pm 0.74$ & 6 \\
\hline & Total & $3.83 \pm 0.51$ & $5.30 \pm 0.53$ & $6.79 \pm 0.62$ & $7.99 \pm 0.50$ & $8.87 \pm 0.43$ & 40 \\
\hline & & & $1.47 \pm 0.39$ & $2.96 \pm 0.54$ & $4.17 \pm 0.49$ & $3.21 \pm 0.70$ & 40 \\
\hline $\begin{array}{l}\mathrm{p} \text {-value (for } \\
\text { raw data) }\end{array}$ & & 0.187 & $<0.001$ & $<0.001$ & $<0.001$ & $<0.001$ & \\
\hline $\begin{array}{c}\mathrm{p} \text {-value } \\
\text { for change } \\
\text { in pupil } \\
\text { diameter }\end{array}$ & & & $<0.001$ & $<0.001$ & $<0.001$ & $<0.001$ & \\
\hline
\end{tabular}

b) Aberrometer

\begin{tabular}{|c|c|c|c|c|c|c|c|}
\hline $\begin{array}{l}\text { Diabetes } \\
\text { Status }\end{array}$ & $\begin{array}{l}\text { Age } \\
\text { Group }\end{array}$ & $\begin{array}{c}\text { 0 Mins } \\
(\text { Mean } \pm \text { SD })\end{array}$ & $\begin{array}{c}15 \text { mins } \\
(M e a n \pm S D)\end{array}$ & $\begin{array}{c}30 \text { mins } \\
(\text { Mean } \pm \text { SD })\end{array}$ & $\begin{array}{c}45 \text { mins } \\
(\text { Mean } \pm \text { SD })\end{array}$ & $\begin{array}{c}60 \text { mins } \\
(M e a n \pm S D)\end{array}$ & $\mathbf{N}$ \\
\hline \multirow{10}{*}{ No diabetes } & $40-49$ & $6.44 \pm 0.57$ & $7.13 \pm 0.37$ & $8.05 \pm 0.52$ & $9.68 \pm 0.19$ & $9.97 \pm 0.07$ & 26 \\
\hline & & & $0.68 \pm 0.72$ & $1.60 \pm 0.81$ & $3.23 \pm 0.56$ & $3.53 \pm 0.58$ & 26 \\
\hline & 50- 59 & $5.89 \pm 0.88$ & $7.20 \pm 0.18$ & $7.84 \pm 0.28$ & $9.49 \pm 0.28$ & $10.00 \pm 0.00$ & 10 \\
\hline & & & $1.31 \pm 0.87$ & $1.95 \pm 0.97$ & $3.60 \pm 1.04$ & $4.11 \pm 0.88$ & 10 \\
\hline & $60-69$ & $5.15 \pm 0.10$ & $7.02 \pm 0.12$ & $7.68 \pm 0.15$ & $9.22 \pm 0.08$ & $9.93 \pm 0.12$ & 3 \\
\hline & & & $1.87 \pm 0.21$ & $2.53 \pm 0.23$ & $4.07 \pm 0.16$ & $4.78 \pm 0.21$ & 3 \\
\hline & $70-79$ & $5.30 \pm 0.00$ & $6.90 \pm 0.00$ & $7.30 \pm 0.00$ & $8.80 \pm 0.00$ & $9.40 \pm 0.00$ & 1 \\
\hline & & & $1.60 \pm 0.00$ & $2.00 \pm 0.00$ & $3.50 \pm 0.00$ & $4.10 \pm 0.00$ & 1 \\
\hline & Total & $6.18 \pm 0.74$ & $7.13 \pm 0.32$ & $7.95 \pm 0.46$ & $9.58 \pm 0.28$ & $9.96 \pm 0.11$ & 40 \\
\hline & & & $0.95 \pm 0.81$ & $1.77 \pm 0.85$ & $3.40 \pm 0.72$ & $3.78 \pm 0.74$ & 40 \\
\hline \multirow{10}{*}{$\begin{array}{l}\text { Diabetes } \\
\text { without } \\
\text { retinopathy }\end{array}$} & $40-49$ & $6.18 \pm 0.50$ & $7.07 \pm 0.17$ & $7.65 \pm 0.28$ & $8.70 \pm 0.34$ & $9.60 \pm 0.39$ & 5 \\
\hline & & & $0.89 \pm 0.51$ & $1.47 \pm 0.37$ & $2.52 \pm 0.40$ & $3.52 \pm 0.40$ & 5 \\
\hline & $50-59$ & $6.31 \pm 0.35$ & $7.07 \pm 0.41$ & $7.79 \pm 0.27$ & $8.71 \pm 0.27$ & $9.60 \pm 0.14$ & 15 \\
\hline & & & $0.76 \pm 0.38$ & $1.48 \pm 0.28$ & $2.40 \pm 0.26$ & $3.29 \pm 0.37$ & 15 \\
\hline & $60-69$ & $6.04 \pm 0.56$ & $6.97 \pm 0.41$ & $7.45 \pm 0.35$ & $8.46 \pm 0.28$ & $9.50 \pm 0.30$ & 14 \\
\hline & & & $0.94 \pm 0.51$ & $1.41 \pm 0.52$ & $2.42 \pm 0.45$ & $3.46 \pm 0.37$ & 14 \\
\hline & $70-79$ & $5.48 \pm 0.18$ & $6.61 \pm 0.32$ & $7.19 \pm 0.21$ & $8.23 \pm 0.34$ & $9.42 \pm 0.17$ & 6 \\
\hline & & & $0.82 \pm 0.10$ & $1.72 \pm 0.19$ & $2.75 \pm 0.37$ & $3.94 \pm 0.20$ & 6 \\
\hline & Total & $6.07 \pm 0.51$ & $6.97 \pm 0.40$ & $7.56 \pm 0.36$ & $8.55 \pm 0.33$ & $9.54 \pm 0.25$ & 40 \\
\hline & & & $0.90 \pm 0.44$ & $1.49 \pm 0.38$ & $2.48 \pm 0.37$ & $3.47 \pm 0.40$ & 40 \\
\hline
\end{tabular}




\begin{tabular}{|c|c|c|c|c|c|c|c|}
\hline \multirow{10}{*}{$\begin{array}{l}\text { Diabetes } \\
\text { retinopathy }\end{array}$} & $40-49$ & $5.88 \pm 0.44$ & $6.44 \pm 0.42$ & $7.38 \pm 0.38$ & $8.11 \pm 0.51$ & $8.93 \pm 0.46$ & 6 \\
\hline & & & $0.58 \pm 0.52$ & $1.50 \pm 0.50$ & $2.23 \pm 0.44$ & $3.05 \pm 0.35$ & 6 \\
\hline & $50-59$ & $5.84 \pm 0.62$ & $6.52 \pm 0.47$ & $7.38 \pm 0.46$ & $8.05 \pm 0.63$ & $8.85 \pm 0.57$ & 13 \\
\hline & & & $0.68 \pm 0.82$ & $1.53 \pm 0.77$ & $2.21 \pm 1.09$ & $3.01 \pm 0.96$ & 13 \\
\hline & $60-69$ & $5.45 \pm 0.51$ & $6.84 \pm 0.56$ & $7.41 \pm 0.71$ & $8.21 \pm 0.39$ & $9.03 \pm 0.33$ & 15 \\
\hline & & & $1.39 \pm 0.60$ & $1.96 \pm 0.80$ & $2.76 \pm 0.59$ & $3.58 \pm 0.53$ & 15 \\
\hline & $70-79$ & $5.93 \pm 0.38$ & $6.75 \pm 1.04$ & $7.21 \pm 0.21$ & $7.98 \pm 0.43$ & $8.82 \pm 0.31$ & 6 \\
\hline & & & $0.82 \pm 1.00$ & $1.28 \pm 0.46$ & $2.05 \pm 0.38$ & $2.88 \pm 0.23$ & 6 \\
\hline & Total & $5.71 \pm 0.55$ & $6.66 \pm 0.61$ & $7.36 \pm 0.53$ & $8.11 \pm 0.49$ & $8.92 \pm 0.43$ & 40 \\
\hline & & & $0.95 \pm 0.79$ & $1.65 \pm 0.74$ & $2.40 \pm 0.79$ & $3.21 \pm 0.70$ & 40 \\
\hline $\begin{array}{l}\mathrm{p} \text {-value (for } \\
\text { raw data) }\end{array}$ & & 0.146 & 0.007 & 0.041 & $<0.001$ & $<0.001$ & \\
\hline $\begin{array}{l}\mathrm{p} \text {-values } \\
\text { for change } \\
\text { in pupil } \\
\text { diameter }\end{array}$ & & & 0.077 & 0.041 & $<0.001$ & $<0.001$ & \\
\hline
\end{tabular}

Table 2: Mean pupil diameter as well as mean change (from baseline) in pupil diameter (boldface) for each of the study groups and the respective age groups as measured with (a) Orbscanand (b) Aberrometer.

\begin{tabular}{|c|c|c|c|c|c|}
\hline Instrument & $\begin{array}{l}\text { Time after } \\
\text { instillation of } \\
\text { drops (min) }\end{array}$ & $\begin{array}{l}\text { Percentage variation } \\
\text { in pupil size due to } \\
\text { difference in diabetic } \\
\text { status }\left(h^{2} \times 100\right)\end{array}$ & $\begin{array}{l}\text { Percentage variation } \\
\text { in pupil size due to } \\
\text { difference in age }\left(h^{2} x\right. \\
100)\end{array}$ & $\begin{array}{l}\text { p-value for effect of } \\
\text { diabetic status }\end{array}$ & $\begin{array}{l}\text { p-value for } \\
\text { effect of } \\
\text { age }\end{array}$ \\
\hline \multirow{9}{*}{ Orbscan } & Baseline (0 min) & 3.1 & 19.4 & 0.187 & $<0.001$ \\
\hline & 15 & 25.7 & 10.8 & $<0.001$ & 0.006 \\
\hline & & 20.6 & 6.2 & $<0.001$ & 0.062 \\
\hline & 30 & 24.9 & 11.9 & $<0.001$ & 0.003 \\
\hline & & 23 & 5.7 & $<0.001$ & 0.081 \\
\hline & 45 & 55.7 & 17.3 & $<0.001$ & $<0.001$ \\
\hline & & 51.1 & 3.2 & $<0.001$ & 0.293 \\
\hline & 60 & 57.5 & 8.6 & $<0.001$ & 0.002 \\
\hline & & 33.2 & 12.8 & $<0.001$ & 0.001 \\
\hline \multirow{9}{*}{ Aberrometer } & Baseline $(0 \mathrm{~min})$ & 3.5 & 14 & 0.146 & 0.001 \\
\hline & 15 & 8.7 & 1 & 0.007 & 0.782 \\
\hline & & 4.4 & 13.1 & 0.077 & 0.001 \\
\hline & 30 & 5.7 & 6.2 & 0.041 & 0.073 \\
\hline & & 5.5 & 4.4 & 0.041 & 0.164 \\
\hline & 45 & 47.1 & 9.5 & $<0.001$ & 0.013 \\
\hline & & 34 & 5.4 & $<0.001$ & 0.094 \\
\hline & 60 & 57.7 & 8.6 & $<0.001$ & 0.02 \\
\hline & & 21 & 11.8 & $<0.001$ & 0.002 \\
\hline
\end{tabular}

Table 3: Variation in pupil size and mean change in pupil size (boldface) due to differences in diabetes status and age as well as the respective $\mathrm{p}$-values for the effects. 


\section{Open Access Journal of Ophthalmology}

A $3 \times 4$ (diabetes status $\mathrm{x}$ age group) factorial ANOVA indicated that there was a significant difference in the mean change in pupil diameter for the three groups $(p<0.001)$ for Orbscan at all the time intervals after instillation of dilating agent. However, with the Aberrometer, there was a significance difference in the change in pupil diameter among the three groups at 30, 45 and 60 minutes post instillation of mydriatic drops $(\mathrm{p}<0.05)$ (Table 2). Table 3 also shows that the effect of diabetic status increased from $20.6 \%$ at 15 minutes to reach a peak of $51.1 \%$ at 45 minutes post instillation of mydriatic drops for Orbscan while for Aberrometer, it was $4.4 \%$ to reach a peak of $34 \%$ over the same time interval.

\section{Discussion}

We investigated the effect of combined phenylephrine and tropicamide eye drops on pupillary dilatation with the aim of using the difference in pupil dilatation on diabetic and non-diabetic patients as a marker for diabetes mellitus. We also compared pupil dilatation in diabetic patients with retinopathy and without retinopathy.

The $3 \times 4$ (diabetes status $x$ age group) factorial analysis of variance (ANOVA) indicated that there was a significant difference in pupil diameter among the three study groups at 15 mins post-instillation of the mydriatic agent which persisted up to the 60 minutes post-instillation irrespective of the instrument used to measure pupil diameter. However, post-hoc indicated no significant difference at baseline between the non-diabetic and the diabetic group with a significant difference only noted between diabetics and diabetic retinopathy group irrespective of the instrument used. As the mydriasis progressed a significant difference was noted among the three groups. The differences in diabetic status (non-diabetics, diabetics and diabetic retinopathy groups) accounted for variation in pupil diameter to different degrees (Table 3). It was observed that at $45 \mathrm{mins}$ after the instillation of the mydriatic drops, differences in diabetic status accounted for $55.7 \%$ and $47.1 \%$ when measured with Orbscan and Aberrometer respectively while the corresponding effect of age was $17.3 \%$ and $9.5 \%$ for each respective instrument.

Similarly, there was a significant difference in the change in pupil diameter relative to baseline at all-time interval for both instruments except for the first 15 minutes with Aberrometer (Table 2). The peak effect was also notice at 45 minutes with diabetic status accounting for $51.1 \%$ and $34.0 \%$ of the change in pupil diameter for Orbscan and Aberrometer respectively.

Studies have reported a significant difference in pupil diameter between diabetics and non-diabetic controls with the reported change occurring at 40 mins after instillation of mydriatic eye drop $[13,19]$. However, the findings from this study cannot be compared with the study [19] that reported differences in pupil diameter at 40 minutes post-instillation of mydriatic eye drops because of the different instrument used in measuring pupil diameter. Notwithstanding the difference in the instrument used in measuring pupil diameter, it can be concluded that this variation exists as we also observed this difference irrespective of whether the Orbscan or Aberrometer was used in pupil diameter measurement. Studies $[13,23]$ have reported reduced pupil diameter in children and adolescents with diabetes compared with their non-diabetic counterparts with Karavanakiand coworkers reporting a weak negative correlation with duration of diabetes [13]. Significant differences in baseline pupil diameter have also been reported in adults [24]. Similar significant difference in the pupil diameter of diabetic and non-diabetic controls have been reported [25], with the later having larger pupil diameter. In the present study, there was also a significant difference in the pupil diameter of diabetic and non-diabetic patients at $15 \mathrm{mins}, 30 \mathrm{mins}$, 45 mins and 60 mins post dilatation (Table 2), consistent with the results of previous studies. Whereas previous studies examined variation in pupil diameter between diabetics and non-diabetics at a specific time interval, the present study evaluated the pupil diameter among nondiabetics and diabetics with and without retinopathy as well as at 15 minutes interval for one hour post instillation of mydriatic agent. From the post-hoc analysis, the difference in pupil diameter was not significant at baseline but became significant as mydriasis progressed.

In contrast to the previous [19,23-25] and the present study, Coblentz J, et al. [20] did not report the difference in mean pupillary diameter between diabetics and nondiabetics. This could be due to the difference in the pharmacological protocol used. In the present study, $0.8 \%$ tropicamide and $5 \%$ phenylephrine without pre-instillation of anesthetics to achieve mydriasis whereas Coblentz J, et al. [20] used $10 \%$ phenylephrine and $1 \%$ tropicamide with pre-instillation of $0.5 \%$ proximetacaine (local anesthetics) to achieve mydriasis.

The result showed that non-diabetics have larger pupil diameter compared to diabetics with or without retinopathy, with the retinopathy group consistently having lower pupil size at baseline and at each time interval after instillation of mydriatic agent (Table 2). This finding is consistent with the result of previous report that non-diabetics have larger pupils compared to diabetic patients [26]. In the present study, we further observed that non-diabetics have the largest pupil diameter before and during dilation followed by diabetics without retinopathy and the retinopathy group having the least pupil diameter. This trend was present irrespective of the 


\section{Open Access Journal of Ophthalmology}

instrument used. Furthermore, the change in pupil diameter from baseline values also indicated a similar decrease with respect to the diabetes status. The non-diabetic groups had the largest change followed by the diabetic groups without retinopathy while the diabetic retinopathy group showed the least change in pupil diameter from baseline. With the Orbscan, the changes in pupil diameter with respect to the three groups were significant at 15, 30, 45 and 60 minutes from baseline. Significant change in pupil diameter was only recorded at 30, 45 and 60 minutes from baseline with the Aberrometer (Table 2). Whereas the effect of the diabetes status on the change in pupil diameter from baseline was significant at the different time intervals for Orbscan and at 30, 45 and 60 minutes for Aberrometer, the effect of age was only significant at 60 minutes from baseline with Orbscan and at 15 and 60 minutes from baseline for Aberrometer (Table 3 ). In any case, the percentage variation due to difference in diabetic status was as much as $51.1 \%$ for Orbscan and $34.0 \%$ for Aberrometer with both effects achieved at 45 minutes from baseline while that due to age was consistently less than $15 \%$ and less than the variation due to diabetic status except at 15 minutes for Aberrometer (Table 3).

From the results, it is obvious that diabetic status does affect pupil diameter and the response to mydriatic agent with the maximum effect being attained at 45 minutes following instillation of mydriatic agent.

Our study shows that at 45 minutes after installation of first drop of Tropicamide and phenylephrine, the pupil size was larger than the pupil size of $7.74 \mathrm{~mm}$ in nondiabetic and $7.23 \mathrm{~mm}$ in diabetic patients 40 minutes after the instillation of mydriatic agent that has been previously reported [19]. This difference could be due to the differences in the methods of measuring pupil diameter. The pupil gauge (manual method) was used to determine the pupil diameter in the study George A, et al. [19] whereas the current study used the Orbscan and Aberrometer. These instruments give objective measurements of pupil diameter and might be more accurate than the manual pupil gauge. Manual measurement of pupil diameter may underestimate the true pupil diameter because it actually measures the diameter of the exit pupil

With both the Orbscan and Aberrometer, the largest mean difference in pupil diameter in diabetic and nodiabetic patients was recorded at 45 minutes after initiation of mydriasis $(1.31 \mathrm{~mm}$ and $1.26 \mathrm{~mm}$ respectively). Aberrometer gave a higher pupil diameter than the Orbscan. This difference was significant at baseline and at 15 and 30 mins $(p<0.0001)$ after instillation of mydriatic eye drop but it was not significant at $45 \mathrm{mins}(\mathrm{p}=0.111)$ and $60 \mathrm{mins}$ post- instillation $(\mathrm{p}=0.822)$. This difference is expected because of the difference lighting operations used by the two instruments to measure pupil diameter. The Orbscan uses white light while the Aberrometer uses infrared light for the measurement. Differences have been reported when the Orbscan and Aberrometer were used to measure pupil size [26].

Usually diabetic retinopathy occurs in patients with longstanding diabetes or uncontrolled blood sugar. Pupil diameter measured by Orbscan and Aberrometer shows a significant difference $(\mathrm{p}<0.0001)$ in mean values between non retinopathy diabetic subjects and diabetic retinopathy subjects at all intervals after installation of drops. These findings further suggest that reduction in pupil diameter in majority of diabetes subjects may be an indicator of development of diabetic retinopathy with uncontrolled blood sugar levels or long standing diabetes. With both the Orbscan and Aberrometer, the largest mean difference in pupil diameter in diabetic patients with and without diabetic retinopathy was recorded at 60 minutes after initiation of mydriasis $(0.64 \mathrm{~mm}$ and $0.65 \mathrm{~mm}$ respectively).

The duration of diabetes in this study ranged between 0.1 to 27 years with a mean duration of 7.73 years. This is similar to the mean duration of 7.10 years previously reported George A, et al. [19] but different from the range of 0.4 to 13.9 years (median $=4$ years) children and adolescents with diabetes [13]. In the present study, we noted a significant but moderate negative correlation between duration of diabetes and pupil diameter using both the Orbscan and Aberrometer ( $\mathrm{r}=-0.480$ and -0.353 respectively, $\mathrm{p}<0.001$ ). This was consistent with previous studies Karavanaki K, et al. [13], George A, et al. [19] which has also reported an inverse relationship between pupil dilatation and duration of diabetes.

In the present study, there was a significant difference among the non-diabetic, diabetic and diabetics with retinopathy subjects $(p<0.001)$ with the non-diabetics significantly being the youngest and the diabetics with retinopathy being the oldest. Although pupil diameter has been reported to decrease with age, we observed that the difference in pupil size among the three study groups was more due to the effect of the differences in the diabetic status than the age as can be seen from (Table 3). At baseline where the effect of age exceeds the effect of difference in diabetic status, there was no significant difference in pupil size among the three groups. Post-instillation of the mydriatic drops when the variation of pupil size became significant, the effect of variation in diabetic status exceeded the effect of age. The observation that differences in diabetic status may play a role in the variation in pupil size among the three groups, we performed analysis of covariance (ANCOVA) to control for the effect of age. It was observed that after controlling for age, the variation in pupil size among the study groups was significant at all-time intervals except at baseline. 


\section{Open Access Journal of Ophthalmology}

Furthermore, our study revealed a significant but low to moderate negative correlation between age and pupil diameter at baseline and at the different time intervals after initiation of mydriasis. Notwithstanding, the study also noted difference in the pupillary diameter between diabetic patients with and without retinopathy despite both groups having similar mean age.

The poor pupillary dilatation amongst diabetic patients could relate to the autonomic neuropathy associated with diabetes. The dilator muscles of the iris which is supplied by the sympathetic branch of the autonomic nervous system and responsible for pupillary dilatation could have been affected by the autonomic neuropathy in diabetes resulting in reduced action. Furthermore, glycogen has been reported to be deposited in the pigment epithelium of the iris Smith ME, et al. [27], Yanoff M, et al. [28] and these have been suggested to be responsible for poor pupil dilatation in diabetic patients [25].

In spite of the insights provided by this study, there are certain limitations that need to be mentioned. In the present study, the diabetic status of the subjects was made without consideration of the HbA1c status. The fact that the age of the diabetic and non-diabetic subjects were not matched could have influenced the difference in pupil diameter between the two groups examined. The relatively low to moderate negative correlation between age and pupil diameter coupled with difference in the magnitude of the effect of diabetic status and age group on pupil diameter in the factorial ANOVA indicated that diabetic status was more accountable for the variation in pupil size than age. Furthermore, studies that used comparable age groups have reported differences in pupil diameter between diabetic and non-diabetic patients indicating that the influence of age on pupil diameter between the two groups may be minimal.

From our study we have demonstrated that there is reduced pupillary dilatation in diabetic subjects compared to non-diabetic subjects. Noting this reduced pupillary dilatation in individuals who present for eye examination could be used as a signal to further investigate for diabetes. Through additional history to elicit symptoms of diabetes, routine pupil dilatation during regular eye examination could be used to triage for diabetes in previously undiagnosed diabetes. Notwithstanding the results of this study, further studies from different population might be needed to ensure the utility of pupillary dilation as a marker for diabetes.

\section{Acknowledgements}

We would like to acknowledge the contribution of Dr. Pavan Verikicharla from L.V.Prasad Eye Institute, Hyderabad, India for his valuble comments to improve the manuscript and Mr. Madhubalan Joseph and Mr. Sonet Sojan from Lotus Eye care Hospital, Coimbatore, India for their contribution in data collection.

\section{References}

1. Unger J, White R (2016) Diabetes mellitus. In: Rakel RE, et al. (Eds.), Textbook of family medicine. $9^{\text {th }}$ (Edn.), Philadelphia: Elsevier Health Science, pp: 782-815.

2. Danaei G, Finucane MM, Lu Y, Singh MG, Cowan JM, et al. (2011) National, regional, and global trends in fasting plasma glucose and diabetes prevalence since 1980: systematic analysis of health examination surveys and epidemiological studies with 370 country-years and 2.7 million participants. Lancet 378(9785): 31-40.

3. Tuomilehtu J, Lindstrom J, Ericksson JG, Valle TT, Hamalainen $\mathrm{H}$, et al. (2001) Prevention of type 2 diabetes mellitus by changes in lifestyle among subjects with impaired glucos tolerance. N Engl J Med 344: 1343-1350.

4. Omar MA, Seedat MA, Motala AA, Dyer BR, Becker P (1993) The prevalence of diabetes mellitus and impaired glucose tolerance in a group of urban South African Blacks. S Afr Med J 83(9): 641-643.

5. (2007) Centers for Disease Control and Prevention. National diabetes fact sheet: general information and national estimates on diabetes in the United States. Atlanta, GA: US Department of Health and Human Services, Center for Disease and Control and Prevention.

6. Williams R, Gaal LV, Lucioni C (2002) Assessing the impact of complications on the costs of type II diabetes. Diabetologia 45(7): S13-S17.

7. Jeganathan VS, Wang JJ, Wong TY (2008) Ocular association of diabetes other than diabetic retinopathy. Diabetic Care 31(9): 1905-1912.

8. Meigs J (2009) Multiple biomarker predict Type 2 diabetes. Diabetes Care 32(7): 1346-1348.

9. Khansari MM, Wanek J, Tan M, Joslin CE, Kresovich KJ, et al. (2007) Assessment of conjunctival microvascular hemodynamics in stages of diabetic microvasculopathy. Sci Reports 7: 1-8.

10. Phillips KC, Clarke Farr PC, Matsha TE, Meyer D (2018) Biomarkers as a predictor for diabetic retinopathy risk and management: A review. Afr Vis Eye Health 77(1): $1-5$.

11. Dorcely B, Katz K, Jagannathan R, Chiang SS, Oluwadare B, et al. (2017) Novel biomarkers for prediabetes, diabetes, 


\section{Open Access Journal of Ophthalmology}

and associated complications. Diabetes, Metabolism Syndrome and Obesity: Targets and Therapy 10: 345361.

12. Jin J, Min H, Kim SJ, Oh S, Kim K, et al. (2016) Development of diagnostic biomarkers for detecting diabetic retinopathy at early stages using quantitative proteomics. J Diab Res pp: 1-22.

13. Karavanaki K, Davies AG, Hunt LP, Morgan MH, Baum DJ (1994) Pupil size in diabetes. Arch Dis Childhood 71(6): 511-515.

14. Cunha Vaz J (2014) Phenotypes and biomarkers of diabetic retinopathy. Personalized medicine for diabetic retinopathy: the Weisenfeld award. Invest Opthalmol Vis Sci 55(8): 5412-5419.

15. Vujosevic S, Simo R (2017) Local and systemic inflammatory biomarkers of diabetic retinopathy: an integrative approach. Invest Ophthalmol Vis Sci 58(6): BI068-BI075.

16. Blair NP, Wanek J, Felder AE, Joslin EC, Kresovich KJ, et al. (2017) Retinal oximetry and vessel diameter measurement with a commercially available scanning laser ophthalmoscope in diabetic retinopathy. Invest Ophthalmol Vis Sci 58(12): 5556-5563.

17. Jenkins AJ, Joglekar MV, Hardikar AA, Keech CA, Neal OND, et al. (2015) Biomarkers in diabetic retinopathy. Rev Diabet Stud 12(2):159-195.

18. Lyons TJ, Basu A (2015) Biomarkers in diabetes: hemoglobin A1c, vascular and tissue markers. Transl Res 159(4): 303-312.

19. George A, Antony J, Thampi B (2014) Comparison of mydriasis obtained by tropicamide and phenylephrine in type 2 diabetics and non-diabetice patients. Int J Prev
Ther Med 2: 1-3.

20. Coblentz J, Motta MM, Fernades BF, Burnier NM, Vianna GNR (2009) Comparison between obtained mydriasis in type 2 diabetics and non-diabetic patients. Curr Eye Res 34(11): 925-927.

21. Hammond CJ, Snieder H, Spector TD, Gilbert EC (2000) Factors affecting pupil size after dilatation: the Twin Eye Study. Br J Ophthalmol 84(10): 1173-1176.

22. Kankipati L, Girkin CA, Gamlin PD (2011) The PostIllumination Pupil Response Is Reduced in Glaucoma Patients. Invest Ophthalmol Vis Sci 52(5): 2287-2292.

23. Clarke CF, Piesowicz, PT, Spathis CS (1989) Pupillary size in children and adolescents with type I diabetes. Diabet Med 6(9): 780-783.

24. Park JC, Chen Y-F, Blair NP, Chau YF, Lim IJ, et al. (2017) Pupillary responses in non-proliefrative diabetic retinopathy. Sci Reports pp: 1-10.

25. Hreidarsson AB (1982) Pupil size in insulin-dependent diabetes. Relationship to duration, metabolic control, and long-term manifestations. Diabetes 31(5): 442-448.

26. Kohnen T, Terzi E, Kasper T, Kohnen ME, Buhren J (2004) Correlation of infrared pupillometers and CCD-camera imaging from aberrometry and videokeratography for determining scotopic pupil size. J Cataract Refract Surg 30(10): 2116-2123.

27. Smith ME, Glickman P (1975) Diabetic vacoulation of the iris pigment epithelium. Am J Ophthalmol 79(5): 875877.

28. Yanoff M, Fine BS, Berkow JW (1970) Diabetic lacy vacoulation of iris pigment epithelium. Am J Ophthalmol 69(2): 201-210.

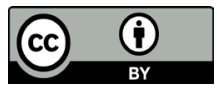

